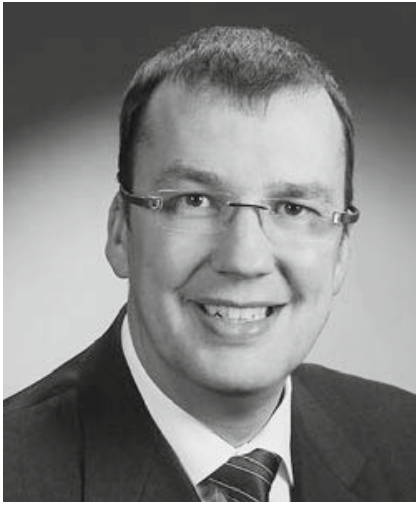

Utz Schäffer

\section{Controlling in China}

\section{Liebe Leser,}

China! Auf wen hat dieses Wort nicht schon als Kind eine enorme Faszination ausgeübt? Waren wir damals von der Großen Mauer, der Verbotenen Stadt, den Reiseberichten Marco Polos und der schieren Größe des fremdartigen Vielvölkerreiches fasziniert, so hat uns in den letzten 30 Jahren zunehmend auch die wirtschaftliche Entwicklung Chinas Respekt abverlangt. Ich selbst durfte in den letzen Jahren mehrere Wochen in Shanghai, Hongkong und dem Perlflussdelta verbringen und fand dabei die alte Regel bestätigt, dass man vieles hören und lesen kann, manches - wie die Dynamik dieser Wirtschaftszentren - aber einfach vor Ort erleben muss.

Mit dem vorliegenden Heft können wir somit auch nur einen ersten Einstieg in die Thematik geben. Ich hoffe aber, dass wir Ihnen mit insgesamt fünf Beiträgen und der bewährten Leseliste einige wertvolle Einsichten zum Controlling in China vermitteln können. Wir haben uns dabei auf die deutsche Perspektive beschränkt: Haben also gefragt, welche Erfahrungen deutsche Unternehmen gesammelt haben und welche Fallstricke auf den unbedarften Controller aus dem Hauptquartier in Deutschland warten.

Wir starten bewusst mit einer chinesischen Perspektive: Im ersten Beitrag stellen Wang Xuyi und Chu Hui vom chinesisch-deutschen Hochschulkolleg der Tongji Universität Shanghai überzeugend dar, warum deutsche Controllingpraktiken und -systeme in einem chinesischen Umfeld nicht unreflektiert Verwendung finden dürfen. Im Anschluss präsentieren wir Ihnen zwei Erfahrungsberichte deutscher Manager: Marcus Gerlach, Mitglied der Geschäftsführung der KS Aluminiumtechnologie $\mathrm{GmbH}$, teilt mit uns seine Erfahrungen aus dem Controlling in einem deutsch-chinesischen Joint-Venture. Im Anschluss berichtet Peter Voss von der Sinoto $\mathrm{GmbH}$ über seine Erfahrungen in der Beschaffung und über Ansätze, wie Controlling eingesetzt werden kann, um die Herausforderungen im chinesischen Beschaffungsmarkt zu meistern. Moritz Maurus, Procter \& Gamble China, und Jens Hildebrandt, Deutsche Auslandshandelskammer China, stellen die interessanten Ergebnisse einer Erhebung zu Aspekten des Risikomanagements bei 36 deutschen Unternehmen in der chinesischen Provinz Guangdong vor. Ein Transferbeitrag fasst schließlich praxisrelevante Erkenntnisse ausgewählter Beiträge international führender wissenschaftlicher Zeitschriften für Sie zusammen.

Es sei aber nicht verschwiegen, dass wir neben dem Schwerpunkt China auch ein spannendes Interview mit Gerrit Steen - CFO der Fresenius Kabi AG - und drei weitere Beiträge in diesem Heft veröffentlichen können. Ein renommiertes Autorenteam um die Kollegen Gunther Friedl, Burkhard Pedell und Hans-Ulrich Küpper stellt empirische Erkenntnisse zur Kostenrechnung in deutschen Großunternehmen vor und kommt zum Schluss, dass die Nutzer alles in allem mit den Rechnungssystemen sehr zufrieden sind. Jens Grundei und Ludger Becker wenden sich in ihrem Beitrag nicht einem klassischen ControllingInstrument, sondern einem noch weitgehend unerforschten Feld zu: dem Controlling organisationaler Aspekte im Unternehmen. Schließlich diskutieren Ute Vanini und Sebastian Bayer in der Rubrik Wissenschaft, wie die Konzernrechnungslegung nach IFRS und eine interne Profit-Center-Rechnung harmonisiert werden können. Auch in diesem Heft wird somit deutlich, wie breit Controllers' Themenstrauß in der Praxis ist!

Es bleibt zu hoffen, dass die Dynamik der chinesischen Wirtschaft über kurz oder lang der Krise trotzt und damit weiter zum Wohlstand in China und in Deutschland beiträgt. Viel Spaß bei der Lektüre wünscht Ihnen

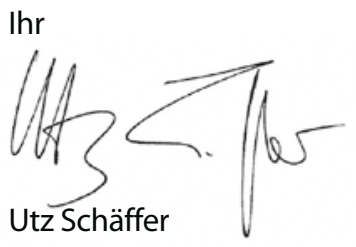

